\title{
Diagnostische Wertigkeit der Röntgenthoraxaufnahme bei tracheobronchialen Fremdkörpern im Kindesalter
}

\author{
Diagnostic Value of a Plain Radiograph of the Chest in Suspected \\ Tracheobronchial Foreign Body Aspiration in Children
}

Autoren

Institute
A. Merkenschlager ${ }^{1}$, L. Sanktjohanser ${ }^{2}$, C. Hundt ${ }^{3}$, K. Schneider ${ }^{3}$, T. Nicolai ${ }^{2}$

Klinik und Poliklinik für Kinder und Jugendliche der Universität Leipzig (Direktor W. Kiess)

2 Haunersches Kinderspital der Universität München (Direktor D. Reinhardt)

3 Abt. für Kinderradiologie, Haunersches Kinderspital der Universität München eingereicht 26.1.2009

akzeptiert $\quad 6.4 .2009$

\section{Bibliografie}

DOI $10.1055 / \mathrm{s}-0029-1214672$

Online-Publikation: 19. 5. 2009

Pneumologie 2009; 63:

325-328 @ Georg Thieme

Verlag KG Stuttgart · New York

ISSN 0934-8387

Korrespondenzadresse

Prof. Dr. Andreas Merkenschlager

Klinik und Poliklinik für Kinder und Jugendliche

Zentrum für Frauen- und

Kindermedizin

Universität Leipzig

Liebigstr. 20a

04103 Leipzig

Andreas.Merkenschlager@

medizin.uni-leipzig.de

\section{Zusammenfassung \\ $\nabla$}

Hintergrund: Über den Einfluss spezifischer radiologischer Erfahrung und der Bildqualität auf den diagnostischen Wert der p.a.-Röntgenthoraxaufnahme bei Verdacht auf einen tracheobronchialen Fremdkörper (FK) im Kindesalter ist wenig bekannt.

Methode und Patienten: 319 p.a.-Röntgenthoraxaufnahmen wurden durch Kinderradiologen, Radiologen in kinderradiologischer Weiterbildung und einen pädiatrischen Facharzt retrospektiv hinsichtlich der Wahrscheinlichkeit eines FK beurteilt (216 Kinder mit bronchoskopisch gesichertem FK, 103 Kinder ohne FK mit Symptomatik vereinbar mit FK).

Ergebnisse: 1) Die Sensitivität war signifikant höher für den Radiologen im Vergleich zu dem Kinderradiologen und dem Pädiater $(70,8 \%$ vs. 59,5 bzw. 56,8\%). Die Spezifität verhielt sich gegenläufig (Radiologe 61,2, Kinderradiologe 73,8, Pädiater 77,7\%). Keine signifikanten Unterschiede bestanden für Korrektheit, positiven und negativen prädiktiven Wert. 2) Technische Mängel wirkten sich nur für den Pädiater, nicht für die radiologischen Spezialisten auf die Beurteilung aus. Durch Exspirationslage wurde die Sensitivität nicht signifikant gesteigert.

Schlussfolgerungen: Auch langjährige spezifisch kinderradiologische Erfahrung erhöht die begrenzte diagnostische Wertigkeit der p.a.-Thoraxaufnahme nicht. Die mangelnde orthograde Einstellung wird durch radiologisches Training offenbar kompensiert, die Atemlage hat unabhängig von der radiologischen Erfahrung keinen Einfluss auf die diagnostische Wertigkeit.

\section{Abstract \\ $\nabla$}

Background: It is not well known how specific experience in paediatric radiology and the radiological technique influence the diagnostic yield of a plain radiograph of the chest when a paediatric foreign body aspiration (FB) is suspected.

Methods and Patients: 319 radiographs were retrospectively evaluated by a paediatric radiologist, a radiologist in paediatric radiology training and an experienced paediatrican with respect to the probability of a tracheobronchial FB (216 children with proven FB, 103 children without FB but with symptoms compatible with FB).

Results: 1) Sensitivity was highest for the radiologist compared to the two other subspecialists (70.8 vs. $59.5 \%$ paediatric radiologist; $56.8 \%$ paediatrican). The specifity was highest for the paediatrican and the paediatric radiologist (77.7 and $73.8 \%$; $62.2 \%$ radiologist). Correctness as well as positive and negative predictive value were not significantly different. 2) The impact of technical quality correlated with the degree of radiological experience, but use of the expiratory phase in general did not increase the sensitivity.

Conclusions: Even profound experience in paediatric radiology does not improve the limited diagnostic value of a plain radiograph of the chest in case of a suspected FB. Radiological training compensates for incorrect patient alignment. An expiratory phase image is not necessary. 


\section{Einleitung}

$\nabla$

Neben Anamnese und Auskultation gilt die Röntgen-Thoraxaufnahme als diagnostisches Standardverfahren bei der Evaluation eines vermuteten tracheobronchialen Fremdkörpers (FK) im Kindesalter [1]. Eine möglichst frühzeitige Diagnose eines FK ist wichtig, um die potenzielle Gefährdung auszuschalten [2] und um sekundäre bronchopulmonale Schäden zu vermeiden [3-7]. Ein bemerktes Erstickungsereignis gilt als anamnestischer Hinweis mit hoher diagnostischer Wertigkeit, die Sensitivität wird mit $97 \%$ bzw. $96 \%$ und die Spezifität mit 63\% bzw. 76\% angegeben $[8,9]$. Allerdings wird ein Aspirationsereignis nur bei etwa $80 \%$ der Kinder beobachtet [10]. Bei etwa einem Fünftel der Kinder muss daher ohne eindeutige anamnestische Hinweise an einen Fremdkörper gedacht und die Indikation zur Bronchoskopie gestellt werden. Dabei ist einerseits zu bedenken, dass auch körperliche Untersuchung in bis zu 20\% der Fälle als unauffällig beschrieben wird [11]. Andererseits sind die auskultatorischen Befunde meist nicht spezifisch und die Zahl möglicher Differenzialdiagnosen groß (z.B. Asthma bronchiale, Tuberkulose oder Bronchitis).

Deshalb ist die diagnostische Wertigkeit der Röntgen-Thorax-Diagnostik bei Verdacht auf tracheobronchialen Fremdkörper im Kindesalter bedeutsam. Dazu wird in der Literatur auf die hohe Inzidenz von unauffälligen Röntgenbefunden hingewiesen $[12,13]$.

Allerdings lassen die bisher publizierten Untersuchungen Fragen offen. Die Röntgen-Thorax-Aufnahme in p.a.-Strahlengang ist die am häufigsten erwähnte radiologische Technik. Dennoch haben sich einige Untersuchungen in der Aussage zur diagnostischen Aussagekraft der Röntgenuntersuchung auf Kombinationen mit der Durchleuchtung bezogen $[14,15]$. Zum anderen waren die Untersuchungen selten so angelegt, dass die Spezifität zuverlässig ermittelt werden konnte. So berichten Zarella u. Mitarb. über 293 prä-bronchoskopische Röntgenuntersuchungen, wobei sich nur in 28 Fällen kein Fremdkörper fand und sämtliche Aufnahmen von Patienten stammten, bei denen eine Bronchoskopie unter FK-Verdacht geplant war [12]. Bereits das klinische Szenario machte hier wegen der hohen Sensitivität des Erstickungsereignisses einen FK hochgradig wahrscheinlich und die Röntgendiagnostik beeinflusste das therapeutische Vorgehen nicht. Auch wenn gezielt Angaben zur Spezifität gemacht werden, so basieren sie meist auf Patienten mit in fast $90 \%$ positiver Aspirationsanamnese und auf relativ kleinen Patientenzahlen $[9,16,17]$.

Gar nicht adressiert wurden bisher die Fragen, ob radiologische bzw. spezifische kinderradiologische Erfahrung des jeweiligen Untersuchers die Aussagekraft beeinflussen und inwieweit radiologische Qualitätsmerkmale hierbei bedeutsam sind. Diese Fragen sind von hoher praktischer Relevanz, weil sie darüber entscheiden könnten, ob Röntgendiagnostik z. B. bei nicht-orthogradem Strahlengang wiederholt werden muss - mit kumulativer Erhöhung der applizierten Strahlendosis - oder ob ein kinderradiologisch besonders erfahrener Gutachter die diagnostische Sicherheit erhöht.

\section{Methode}

$\nabla$

Drei voneinander unabhängige Gutachter beurteilten retrospektiv ohne Kenntnis der Anamnese und klinischer Befunde jeweils die gleichen 319 Röntgenthoraxaufnahmen, angefertigt in p.a.Strahlengang. Die Beurteiler unterschieden sich hinsichtlich ih-
Tab. 1 Übersicht über Sensitivität, Spezifität, Korrektheit, positiven und negativen prädiktiven Wert.

\begin{tabular}{|c|c|c|c|}
\hline Beurteiler & $\begin{array}{l}\text { Kinder- } \\
\text { radiologe }\end{array}$ & Radiologe & Pädiater \\
\hline \multicolumn{4}{|l|}{ Parameter } \\
\hline Sensitivität & $59,5 \%$ & $70,8 \%$ & $56,8 \%$ \\
\hline Spezifität & $73,8 \%$ & $61,2 \%$ & $77,7 \%$ \\
\hline Korrektheit & $64,3 \%$ & $67,6 \%$ & $63,8 \%$ \\
\hline $\begin{array}{l}\text { Positiver prädiktiver } \\
\text { Wert }\end{array}$ & $81,2 \%$ & $78,7 \%$ & $83,6 \%$ \\
\hline $\begin{array}{l}\text { Negativer prädiktiver } \\
\text { Wert }\end{array}$ & $47,8 \%$ & $50,8 \%$ & $47,3 \%$ \\
\hline
\end{tabular}

rer Erfahrung in Radiologie: ein Kinderradiologe (K. S.) hatte 20 Jahre kinderradiologische Berufserfahrung, ein Radiologe (C. H.) in kinderradiologischer Ausbildung 2 Jahre, ein pädiatrischer Facharzt (A. M.) bronchologische Erfahrung ohne spezifisches kinderradiologisches Training.

Die Röntgenbilder stammten 216-mal von Patienten mit bronchoskopisch gesichertem FK. Diese Bilder wurden aus einer Serie von 447 Patienten mit gesicherter FKA zufällig herausgegriffen. 103 Aufnahmen wurden von Kindern ohne FK zugemischt. Diese Aufnahmen waren bei V.a. FK angefertigt worden, der sich schließlich nicht bestätigte (auch kein Nidus bei Bronchoskopie) oder bei einer mit einer FKA vereinbaren Symptomatik.

Die Röntgenthoraxaufnahmen wurden überwiegend mit analoger Technik angefertigt. Im eigenen Hause wurden sie - abhängig vom Gewicht - überwiegend mit folgenden technischen Parametern erstellt: Röhrenspannung $70 \mathrm{kV}$, Fokus-Film-Abstand $150 \mathrm{~cm}$, Röhrenstrom ca. 2 mAs, Dosis ca. 0,4-0,8 cGyxcm², Film-Foliensystem Empfindlichkeitsklasse 400.

Beide Gruppen von Röntgen-Thoraxaufnahmen stammten von Patienten mit analoger Altersstruktur: bei bestätigtem FK Median 21,4 Monate (9-132 Lebensmonate); ohne FK Median 21,1 Lebensmonate (6-144 Lebensmonate). Die Bilder wurden numerisch codiert, Angaben zu Namen und Alter entfernt und zufällig durcheinandergemischt dem Gutachter vorgelegt. Die Gutachter beurteilten die Bilder unabhängig voneinander. Die Auswertungen wurde am gleichen Alternator durchgeführt, damit die Bedingungen für die drei Gutachter gleich waren. Dabei wurde auf eine korrekte Lichtdichte geachtet.

Aufgrund seiner 20-jährigen Erfahrung hatte der Kinderradiologe neben der Wahrscheinlichkeit des Vorliegens eines FK auch zu beurteilen, ob eine Exspirationslage vorlag, eine orthograde Einstellung gegeben war und ein korrekter Schwärzungsgrad erreicht wurde.

Berechnet wurden aus diesen Angaben Sensitivität, Spezifität, Korrektheit, positive und negative prädiktive Potenz der Röntgenthoraxaufnahme und die Beeinflussung dieser Parameter durch etwaige Qualitätsmängel.

\section{Ergebnisse \\ $\nabla$}

\section{Sensitivität}

Die Sensitivität lag für Kinderradiologe, Radiologe in kinderradiologischer Weiterbildung und Pädiater bei 59,5, 70,8 und 56,8\% (s. Tab. 1). Die mittlere Sensitivität lag bei $62 \%$; die 3 Begutachter zeigten bezüglich der Sensitivität einen signifikanten Unterschied ( $\mathrm{p}<0,01$; chi-Quadrat-Test). 


\section{Spezifität}

Die Werte für die Spezifität waren gegenläufig für Kinderradiologen, Radiologen und Pädiater verteilt. Sie betrugen 73,8, 61,2 und 77,7\%. Der Mittelwert der Spezifität lag bei 71\%. Die drei Gutachter unterschieden sich statistisch signifikant $(p=0,02$; chi Quadrat Test).

\section{Korrektheit}

Hier zeigten Kinderradiologe, Radiologe und Pädiater Werte von 64,3, 67,6 und 63,8\%. Der Mittelwert lag bei 65\%; es ergab sich kein statistisch signifikanter Unterschied zwischen den 3 Gutachtern.

\section{Positive Prädiktion}

Die Werte lagen bei $81,2,78,7,83,6 \%$; der Mittelwert lag bei $81 \%$, die Unterschiede waren nicht signifikant.

\section{Negative Prädiktion}

Die Werte für Kinderradiolge, Radiologe und Pädiater lagen bei 47,8, 50,8 und 47,3\%. Die mittlere negative Prädiktion der 3 Gutachter lag bei $49 \%$. Der Unterschied war nicht signifikant.

\section{Qualität der Röntgenthoraxaufnahmen}

Ein korrekter Schwärzungsgrad lag bei 75\% der Aufnahmen (der Kinder mit nachgewiesenem FK) vor ( $n=154)$. Orthograd eingestellt waren $69,4 \%$. Exspirationslage lag nur bei 38,9\% vor. Insgesamt lag eine optimale Bildgüte durch das Zusammentreffen aller 3 Gütekriterien bei 25,5\% der Aufnahmen vor. Gründe für diese Limitationen der Bildqualität lagen darin, dass Fremdaufnahmen nur bei völlig unzureichender Qualität wiederholt wurden oder aufgrund der Altersstruktur schwierige technische Untersuchungsbedingungen gegeben waren.

\section{Einfluss der Qualität auf die Beurteilungs-Sensitivität und -Spezifität}

Der korrekte Schwärzungsgrad hatte bei Kinderradiologe und Radiologe keinen signifikanten Einfluss auf das Verhältnis von richtig-positiven zu falsch-negativen Einschätzungen. Beim Pädiater lagen jedoch immerhin in $84,6 \%$ der richtig-positiven vs. nur $65,2 \%$ der falsch-negativen ein korrekter Schwärzungsgrad vor $(p=0,002)$. Auch die orthograde Einstellung beeinflusste nur beim Pädiater das Verhältnis von richtig-positiv vs. falsch-negativ signifikant ( 81,2 vs. 67,4\%, p =0,04). Die nicht erreichte Exspirationslage war für alle drei ohne signifikanten Einfluss auf die Beurteilung.

\section{Diskussion}

$\nabla$

Die mittlere Sensitivität betrug bei den drei Gutachtern $62 \%$ (56,8-70,8\%). Silva u. Mitarb. geben eine höhere Sensitivität von $73 \%$ an, wobei 82 ihrer 93 Patienten ein Aspirationsereignis in der Anamnese hatten und dadurch ein Trend in der Beurteilung induziert worden sein könnte [16]. Eine Sensitivität von $82 \%$ geben Hoeve et al. an; allerdings handelte es sich hier um Zuweisungen unter dem Verdacht auf FKA (69\%) oder Pneumonie, wobei ebenfalls eine Beeinflussung des Gutachter-Urteils denkbar ist [18].

Andere Untersuchungen berechnen eine Sensitivität von 58,5\% [12], 68\% [17] und 80\% [19]. Die Bandbreite der in der Literatur angegebenen Sensitivität entspricht damit unseren Ergebnissen. Interessanterweise wurde auch bei Kombination von Röntgen-
thorax-Aufnahme mit Thoraxdurchleuchtung keine wesentliche Steigerung der Sensitivität beschrieben [15], wobei andere Autoren eine sehr hohe Sensitivität von Durchleuchtungstechniken angeben - allerdings bei einer Spezifität unter 20\% [20,21]. Auch die seitliche Thoraxaufnahme scheint keine wesentliche diagnostische Zusatzinformation zu bringen [20].

Die mittlere Spezifität betrug 71\% (61,2-77,7\%). Andere Untersuchungen geben deutlich niedrigere Werte für die Spezifität an. Bei Silva et al. liegt diese nur bei $45 \%$, wobei fast alle Patienten eine positive Aspirationsanamnese hatten und die Entscheidung zur Bronchoskopie unabhängig von dem Röntgenbefund war [16]. An einer kleineren Serie von 83 Patienten mit einer FK Prävalenz von $41 \%$ wurde eine Spezifität von $67 \%$ ermittelt [22] und ist damit unseren Daten vergleichbar.

Werte für Sensitivität und Spezifität waren für Kinderradiologe, Radiologen und Pädiater gegenläufig verteilt. Durch die Berechnung der Korrektheit, die für die drei Gutachter bei 64,3, 67,6 und 63,8\% (im Mittel 65,3\%) lag, konnte gezeigt werden, dass Sensitivität und Spezifität sich zueinander umgekehrt proportional verhielten. Waren die 3 Begutachter bezüglich Sensitivität und Spezifität signifikant voneinander different, so zeigten sie hinsichtlich der Korrektheit keinen signifikanten Unterschied. Auch in der positiven und negativen Prädiktion ergab sich kein signifikanter Unterschied. Die Korrektheit und Prädiktion in der Beurteilung scheint also unabhängig von spezifischer radiologischer bzw. kinderradiologischer Erfahrung zu sein.

Die Beurteilung von Sensitivität und Spezifität in Abhängigkeit von der kinderradiologischen Erfahrung ist unseres Wissens nach neu. Dabei ergibt sich für alle drei Gutachter eine vergleichbare diagnostische Korrektheit (accuracy). Dies spricht dafür, dass der radiologischen Erkennung von tracheobronchialen Fremdkörpern Grenzen gesetzt sind und die Korrektheit nicht durch zunehmende, insbesondere kinderradiologische Erfahrung gesteigert werden kann. In der Literatur wird die Röntgendiagnostik meist von einem Radiologen bewertet, nur selten wird explizit mit der Einschätzung einer anderen Fachrichtung verglichen. Zerella et al. tragen der klinischen Realitiät insofern Rechnung, als sie die Spezifität der Röntgendiagnostik in der Bewertung durch den Bronchoskopeur angeben (58,4\%). Sie vergleichen diesen Parameter aber nicht mit einer fachärztlich-radiologischen Beurteilung [12].

Die drei Gutachter unterschieden sich auch nicht signifikant in den Werten der positiven und negativen Prädiktion. Die anhand einer Röntgen-Thorax-Aufnahme in p.a.-Strahlengang gestellte Verdachtsdiagnose einer FKA wäre in $4 / 5$ der Fälle bestätigt worden; wird von radiologischen Kriterien ausgehend ein FK für unwahrscheinlich gehalten, so wird sich bronchoskopisch bei etwa der Hälfte dieser Fälle ein FK finden. Bei alleiniger Indikationsstellung zur Endoskopie durch die p.a.-Röntgenthorax-Aufnahme würden 30-40\% der tracheobronchialen Fremdkörper übersehen werden. Dies bestätigt Angaben der Literatur, wonach die Röntgendiagnostik ein unzuverlässiges Instrument der Diagnostik ist $[9,17,23]$.

In der Literatur fanden wir ebenfalls keine Analyse des Einflusses von technischen Qualitätskriterien auf die Sensitivität. Hierbei hat sich gezeigt, dass die Abhängigkeit von optimaler Bildqualität durch radiologische Erfahrung vermindert wird. So wurde ausschließlich der Gutachter ohne spezifische radiologische Erfahrung in seiner Trefferquote durch mangelnden Schwärzungsgrad und nicht-orthograde Einstellung negativ beeinflusst. So erreicht der Pädiater bei korrekt geschwärzten Aufnahmen eine Sensitivität von $63 \%$, bei nicht korrekt belichteten Aufnahmen nur 36,7\%. 
Die Situation ist für verdrehte Aufnahmen analog. Dabei wird die Bedeutung des Schwärzungsgrades bei Verwendung digitaler Bildverarbeitungssysteme zukünftig weiter an Bedeutung verlieren. Die Daten zeigen zusätzlich, dass die mangelnde Exspirationslage die Beurteilung der Bilder nicht signifikant beeinflusst; somit muss auf die Atemphase bei der Röntgendiagnostik unter dieser Fragestellung kein Wert gelegt werden.

Vor dem Hintergrund der Limitationen der konventionellen Röntgenthorax-Diagnostik ist die Evaluation der Computertomographie (CT) bei FK-Verdacht verständlich; für das CT werden Sensitivitätsangaben von 88,9 bis $100 \%$ gemacht [24-26]. Allerdings ist auch hier die Spezifität z.T. nur gut 60\% [27]. Des weiteren ist bei Durchführung ohne Narkose in über $20 \%$ der Fälle mit erheblichen Artefakten zu rechnen und in bis zu 10\% der Fälle dadurch mit schlechter Bildqualität [24]. Es wird in der Zukunft zu prüfen sein, ob das CT einen Platz in der klinischen Routinediagnostik vor der bei geübter Hand komplikationsarmen Bronchoskopie erhält.

\section{Interessenkonflikt}

$\nabla$

Keine angegeben.

\section{Literatur}

1 Pinto A, Scaglione M, Pinto F et al. Tracheobronchial Aspiration of Foreign Bodies: Current Indications for Emergency Plain Chest Radiography. Radiol Med 2006; 111: 497-506

2 Humphries CT, Wagener JS, Morgan WJ. Fatal Prolonged Foreign Body Aspiration Following an Asymptomatic Interval. Am J Emerg Med 1988; 6: $611-613$

3 Saquib Mallick M, Rauf Khan A, Al Bassam A. Late Presentation of Tracheobronchial Foreign Body Aspiration in Children. J Trop Pediatr 2005; $51: 145-148$

4 Karakoc F, Cakir E, Ersu R et al. Late Diagnosis of Foreign Body Aspiration in Children With Chronic Respiratory Symptoms. Int J Pediatr Otorhinolaryngol 2007; 71: 241-246

5 Linegar AG, von Oppell UO, Hegemann S et al. Tracheobronchial Foreign Bodies. Experience at Red Cross Children's Hospital, 1985-1990. S Afr Med J 1992; 82: 164-167

6 Schimpl G, Weber G, Haberlik A et al. [Foreign Body Aspiration in Children. The Advantages of Emergency Endoscopy and Foreign Body Removal]. Anaesthesist 1991; 40: 479-482

7 Steen KH, During A, Bowing B. X-Ray Signs of Foreign Body Aspiration in Children. Radiologe 1990; 30: 324-327

8 Fontoba JEB, Gutierrez C, Lluna J et al. Bronchial Foreign Body: Should Bronchoscopy Be Performed in All Patients With a Choking Crisis? Pediatr Surg Int 21-3-1997; 12: 118-120
9 Metrangelo S, Monetti C, Meneghini L et al. Eight Years' Experience With Foreign-Body Aspiration in Children: What Is Really Important for a Timely Diagnosis? J Pediatr Surg 1999; 34: 1229-1231

10 Wolach B, Raz A, Weinberg J et al. Aspirated Foreign Bodies in the Respiratory Tract of Children: Eleven Years Experience With 127 Patients. Int J Pediatr Otorhinolaryngol 1994; 30: 1-10

11 Losek JD. Diagnostic Difficulties of Foreign Body Aspiration in Children. Am J Emerg Med 1990; 8: 348 - 350

12 Zerella JT, Dimler M, McGill LC et al. Foreign Body Aspiration in Children: Value of Radiography and Complications of Bronchoscopy. J Pediatr Surg 1998; 33: 1651 - 1654

13 Emir H, Tekant G, Besik C et al. Bronchoscopic Removal of Tracheobroncheal Foreign Bodies: Value of Patient History and Timing. Pediatr Surg Int 2001; 17: 85-87

14 Blazer S, Naveh Y, Friedman A. Foreign Body in the Airway. A Review of 200 Cases. Am J Dis Child 1980; 134: 68-71

$15 \mathrm{Mu}$ LC, Sun DQ He P. Radiological Diagnosis of Aspirated Foreign Bodies in Children: Review of 343 Cases. J Laryngol Otol 1990; 104: 778 - 782

16 Silva AB, Muntz HR, Clary R. Utility of Conventional Radiography in the Diagnosis and Management of Pediatric Airway Foreign Bodies. Ann Otol Rhinol Laryngol 1998; 107: 834-838

17 Svedstrom E, Puhakka H, Kero P. How Accurate Is Chest Radiography in the Diagnosis of Tracheobronchial Foreign Bodies in Children? Pediatr Radiol 1989; 19: 520-552

18 Hoeve LJ, Rombout J, Pot DJ. Foreign Body Aspiration in Children. The Diagnostic Value of Signs, Symptoms and Pre-Operative Examination. Clin Otolaryngol Allied Sci 1993; 18: 55-57

19 Midulla F, Guidi R, Barbato A et al. Foreign Body Aspiration in Children. Pediatr Int 2005; 47: $663-668$

20 Assefa D, Amin N, Stringel G et al. Use of Decubitus Radiographs in the Diagnosis of Foreign Body Aspiration in Young Children. Pediatr Emerg Care 2007; 23: 154-157

21 Ikeda M, Himi K, Yamauchi Yet al. Use of Digital Subtraction Fluoroscopy to Diagnose Radiolucent Aspirated Foreign Bodies in Infants and Children. Int J Pediatr Otorhinolaryngol 1-12-2001; 61: 233-242

22 Svedstrom E, Puhakka H, Kero P. How Accurate Is Chest Radiography in the Diagnosis of Tracheobronchial Foreign Bodies in Children? Pediatr Radiol 1989; 19: 520-522

23 Agarwal RK, Banerjee G, Shembish $N$ et al. Foreign Bodies in the Tracheobronchial Tree: a Review of 102 Cases in Benghazi, Libya. Ann Trop Paediatr 1988; 8: 213-216

24 Kosucu P, Ahmetoglu A, Koramaz I et al. Low-Dose MDCT and Virtual Bronchoscopy in Pediatric Patients With Foreign Body Aspiration. AJR Am J Roentgenol 2004; 183: 1771 - 1777

25 Huang HJ, Fang HY, Chen HC et al. Three-Dimensional Computed Tomography for Detection of Tracheobronchial Foreign Body Aspiration in Children. Pediatr Surg Int 2008; 24: 157-160

26 Kocaoglu M, Bulakbasi N, Soylu Ket al. Thin-Section Axial Multidetector Computed Tomography and Multiplanar Reformatted Imaging of Children With Suspected Foreign-Body Aspiration: Is Virtual Bronchoscopy Overemphasized? Acta Radiol 2006; 47: 746-751

27 Hong SJ, Goo HW, Roh JL. Utility of Spiral and Cine CT Scans in Pediatric Patients Suspected of Aspirating Radiolucent Foreign Bodies. Otolaryngol Head Neck Surg 2008; 138: 576-580 